\title{
Objective correlates of an unusual subjective experience: A single-case study of number-form synaesthesia
}

\author{
M. Piazza and P. Pinel \\ INSERM, CEA, Cognitive Neuroimaging Unit, Orsay, France \\ S. Dehaene \\ INSERM, CEA, Cognitive Neuroimaging Unit, Orsay, France, and Collège de France, Paris, France
}

\begin{abstract}
There is a universal and often unconscious tendency to mentally associate the number sequence with a spatial continuum (the mental number line). Here we study one individual who reports a strong and vivid sense of space when processing numbers. For him, the number sequence has a precise spatial form: a curvilinear right-to-left oriented line. We used various tasks to demonstrate that this numerical-spatial association is not a mere figment of his imagination, but a constrained experiential phenomenon consistent across sessions and automatically triggered by the visual presentation of numbers. We also show that this idiosyncratic representation can coexist with another implicit association, the SNARC effect (Spatial-Numerical Association of Response Codes, where small numbers are associated with the left side of space). This effect is present in individuals without explicit number forms and is not affected in the present subject in spite of his reversed subjective representation.
\end{abstract}

\section{INTRODUCTION}

In 1880 the journal Nature published two articles by the British scientist Sir Francis Galton, entitled "Visualised Numerals", in which he reported a rather intriguing phenomenon: To some otherwise normal people, numbers appeared to occupy very precise locations in space, forming what Galton called "natural lines of thought" (Galton, 1880a, $1880 \mathrm{~b})$. This phenomenon might be considered to be a type of synaesthesia, which is a remarkable condition where an individual has multimodal perceptual experiences, triggered by a unimodal sensory event. Individuals may "taste" shapes, "hear" colours, "feel" sounds, or "see" numbers in space (Baron-Cohen, Wyke, \& Colin, 1987; Ramachandran \& Hubbard, 2001a; Rich \& Mattingley, 2002). Several studies have now approached different types of synaesthetic association in a quantitative fashion and have revealed their "perceptual reality” (Palmeri, Blake, Marois, Flanery, \& Whetsell, 2002; Ramachandran \& Hubbard, 2001a; Smilek, Dixon, Cudahy, \& Merikle, 2001).

Very few investigations, however, have focused on the number-form type of synaesthesia in an experimental fashion (Sagiv, Simner, Collins, Butterworth, \& Ward, in press; Seron, Pesenti, Noël, Deloche, \& Cornet, 1992). Number forms

Correspondence should be addressed to Mauela Piazza, INSERM U562, "Cognitive Neuroimaging", Service Hospitalier Frederic Joliot, 4 Place du general Leclerc, 91401 Orsay cedex, France (E-mail: piazza@shfj.cea.fr).

This study was supported by INSERM, CEA, a Marie Curie fellowship of the European Community QLK6-CT-2002-51635 (M.P.), and a McDonnell Foundation centennial fellowship (S.D.). We would like to thank S.W. for participating in this research and Edward Hubbard and Susannah Revkin for comments on this manuscript. 
are not exceptionally rare. Different estimates of the frequency of number forms in the population range from as little as 6\% (Galton, 1880a; Phillips, 1896) to around 30\% (Calkins, 1892), while the most recent research reports an intermediate frequency of 10-14\% (Sagiv et al., in press; Seron et al., 1992). Interestingly, number forms are more frequent in subjects that also experience grapheme-colour synaesthesia than in subjects that lack any synaesthetic experience (Sagiv et al., in press), suggesting the possibility of a common mechanism between these two different synaesthetic associations.

However, in order to exclude that number forms might simply reflect confabulation, or a particularly rich use of metaphoric language, one needs to be able to objectify the phenomenon by showing that it involves real perceptual experiences, which are stable and reproducible across sessions, and which qualitatively and quantitatively modulate behavioural performance without voluntary control: characteristics that are definitional for synaesthesia (Cytowic, 2002; Sagiv et al., in press).

The purpose of the present study, inspired by similar studies of grapheme-colour synaesthesia (Palmeri et al., 2002; Ramachandran \& Hubbard, 2001a), is to probe whether our subject's number form is a genuine phenomenon and to try to isolate the levels of processing at which numbers and space might interact in number-form synaesthesia.

Moreover, using an approach similar to the one used in neuropsychology, the study of synaesthesia can shed light on normal cognition and here in particular on the more universal association of numbers and space. In fact, even if synaesthesia can be defined with positive rather then negative "symptoms" contrary to most neuropsychological syndromes, it can be described by a set of cognitive characteristics and brain activations that are clearly different from those of the rest of the population (e.g., see E. M. Hubbard, Arman, Ramachandran, \& Boynton, 2005a).

Are the underlying mechanisms of synaesthesia specific to synaesthesia, or are they present in nonsynaesthetes, if perhaps to a lesser degree? Some research suggests that indeed the associations present in at least some kinds of synaesthesia share common mechanisms with nonsynaesthetic associations (e.g., synaesthetic tone-colour associations share some common features with nonsynaesthetic sound pitch and lightness associations; T. L. Hubbard, 1996). Such common mechanisms could account for the "conceptual rightness" of certain spontaneous associations, such as associating an image with round contours with a sound with smooth contours (Köhler, 1920) and may even be at the origin of metaphors in language (Ramachandran \& Hubbard, 2001b). This might indicate that the cross-sensory mappings at the origin of synaesthetic experiences would be present at the neural level in nonsynaesthetes, but would be weaker, so that such cross-modal associations would be strong enough to reach conscious awareness only in synaesthetes (E. M. Hubbard \& Ramachandran, 2005).

Concerning the specific case of number-space associations, it has been shown that the mental representation of numbers is intimately linked with space, even in nonsynaesthetic subjects (Dehaene, 1997). In various tasks, adults from our western society tend to implicitly associate the number sequence with a spatial continuum where small numbers occupy the left and large numbers the right side of space (Dehaene, Bossini, \& Giraux, 1993; Fischer, Castel, Dodd, \& Pratt, 2003). Thus, synaesthetic number forms might represent a conscious and greatly enriched version of a universal tendency to represent numbers in space. Indeed, number forms share two important properties with the "normal" representation of numbers. First, the series of integers is almost always represented by a continuous curve, where consecutive numbers fall one after the other. Second, most synaesthetes report that their number forms become increasingly fuzzier as the numbers increase, either because the number line is seen in 3-D perspective, and therefore large numbers are far away from the observer, or because the line is itself compressed. This is reminiscent of Weber's law and of the postulated logarithmic compression of the internal "number line" (Dehaene, 2002; Nieder \& Miller, 2003; Shepard, Kilpatrick, \& Cunningham, 1975).

Among the many intriguing cases described initially by Galton (1880a) is that of a young man whose number line is shown in Figure 1A. 
A

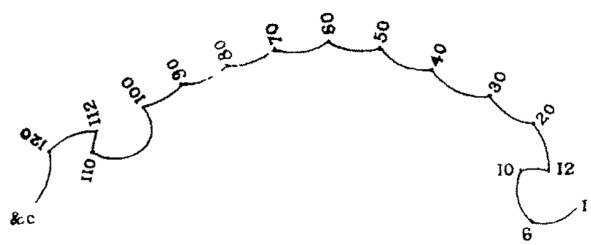

B

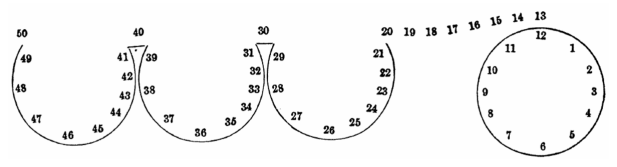

C

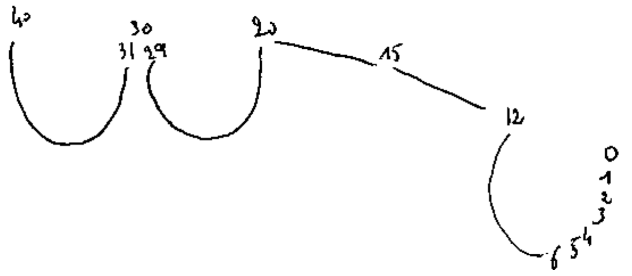

Figure 1. (A) Drawing of the number form of one subject reported from Galton in 1880. (B) Drawing of the number form of one subject reported from Calkins in 1892. (C) Hand drawing of S.W.'s number line.

It is oriented from right to left, starting with a circular form representing the numbers from 1 to 12 , continues with a straight line from 13 to 20, and then progresses in semicircular curves, one for each decade. Cases reporting a very similar form were also later described by Calkins (1892; see Figure 1B) and even more recently by Seron (Seron et al., 1992). More than a hundred years after the first report of this kind, we were amazed to hear from a 32-year-old young man (subject S.W.), a highly educated right-handed music professor who, although he had never read Galton, Calkins, or Seron, or even heard of synaesthesia, reported a virtually identical number form (Figure 1C).

A number form questionnaire (Seron et al., 1992) indicated that S.W. has had this representation ever since he acquired numbers (around age 7). His spatial representation is stable. He claims to be able to see it from different perspectives, depending on which point on the line he places his "point of view", and it is automatically and unavoidably triggered whenever he reads, writes, or speaks a number, whether its meaning is cardinal (quantities, prices), ordinal (dates, times) or, sometimes, denotative (bus numbers, brand names).

S.W. also has a clear form for days of the weeks and hours within the days. Days of the week are represented in a semi-circle, and, linking each day, there are spirals that represent days and nights by circles of 12, and are used to connect one day with the following and the preceding one. Between weeks, S.W. claims that the link between Sunday and Monday "is problematic", as there is no direct connection between the two. His number form also seems to contain annoying incongruent features, as he claims that " 40 is clearly higher than 30 , but at the same time 36 is somewhat slightly lower than 32". He does not report any other kind of synaesthetic association.

In the present study we focus on S.W.'s number form. Our aim is to probe whether S.W.'s number form is a genuine perceptual phenomenon and to try to isolate the levels of processing at which numbers and space might interact.

If we take the perspective proposed by Dixon and colleagues (Dixon, Smilek, \& Merikle, 2004), we would classify S.W. as an "associator" more than a "projector" type of synaesthete, since he described his visuo-spatial experiences as being internal, "in the mind's eye", and not as being "out in the world". Yet according to another possible classification S.W. would fall in the class of "higher" synaesthetes (as opposed to "lower" synaesthetes), in that the inducers of his synaesthetic experience are concepts (numbers, irrespective of the stimulation modality) rather than percepts (Ramachandran \& Hubbard, 2001b).

\section{THE EXPERIMENTS}

\section{Experiment 1: Drawing the number form}

To quantify the consistency of S.W.'s number form, we asked him to reproduce it on a computer screen by placing the mouse cursor on the position of the screen that corresponded to different Arabic numerals. He performed this test twice, the first time in June 2002 and the second time in May 2004. 
We presented several Arabic numerals, one at a time, in white, at the centre of a black screen for $500 \mathrm{~ms}$. There were two blocks, spanning different ranges: In the first block we presented all the odd numbers from 1 to 39 , while in the second we presented all the numbers from 20 to 35 (S.W. was informed of these ranges prior to the testing session). In both blocks each digit was presented 11 times, in random order.

S.W. placed the numbers at screen locations conforming to his previous hand drawings (Figure 2). The error bars show that his report was quite precise and that it was extremely reproducible across a two-year interval (compare Figures 2A and 2B).

\section{Experiment 2: Comparison of numbers to an internal reference}

On average, it takes longer to compare two numerically close numbers than two numerically far numbers (Dehaene, Dupoux, \& Mehler, 1990; Moyer \& Landauer, 1967). We investigated whether S.W.'s number form led to a change in the distance effect. We reasoned that if the
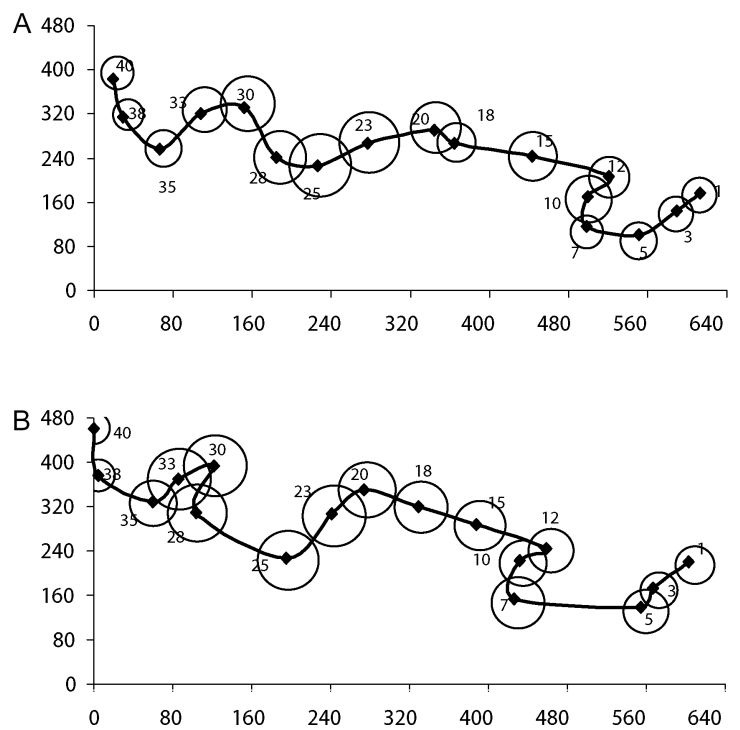

distance effect is generated at the level of a spatial representation of numbers, then S.W.'s judgements might be influenced by the physical straight-line distance between pairs of numbers on his number form. S.W.'s performance was compared to the performance of a control group of 10 right-handed university students naive with regards to the objectives of the present research. Informal questioning at the end of the experiments assessed that none of them experienced number forms. The experiment consisted in comparing different two-digit Arabic numerals to an internal reference, which was fixed at 28. That reference was selected because it is close to a kink in S.W.'s number form (see Figure 1) and hence helped decorrelate numerical and geometrical distance (see below). Each number from 23 to 33 was presented 20 times each, at the centre of the screen, one at a time, for 200 ms. Fillers were added in order to enlarge the range of possible test stimuli (numbers 18 to 22 and 34 to 38 , each presented twice). Participants performed two blocks. In one block, they were asked to respond by pressing with their left hand if the number was smaller than 28 and with the right
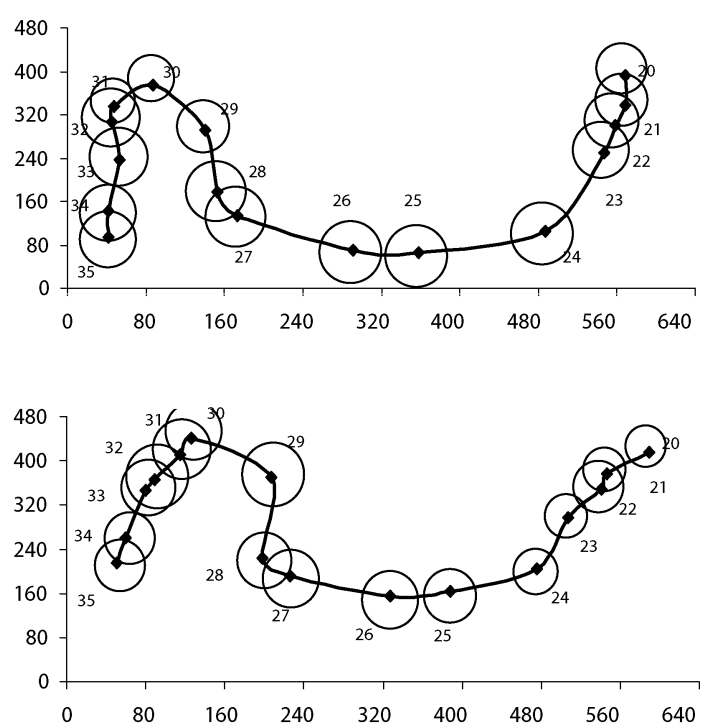

Figure 2. Subject S.W.'s positions of the different numbers on the screen. (A) First session, in June 2002. (B) Second session, May 2004. Left panel, range from 1 to 39. Right panel, numbers from 20 to 35. The axes values are pixels. The diameter of the circle is a measure of the variability of S.W.' judgement within sessions for each tested number, as it is the mean between the standard errors of the positions on the $\mathrm{x}$ and on the $\mathrm{y}$ axes. 
one if it was larger, and in the following block the instructions were reversed. The order of these instructions was given as such to S.W. and was counterbalanced across subjects.

S.W. was extremely accurate $(100 \%$ correct responses) and fast (mean reaction times, $\mathrm{RTs}=$ $614 \mathrm{~ms}$ ). However, neither accuracy nor RTs differed from the controls' performance, for any of the 10 numbers analysed (Figure 2; in all cases $p>$.34). Here and elsewhere we used a onetailed significance test (ST) for comparing individual scores to a small normative sample (Crawford \& Howell, 1998). S.W.'s RTs showed a "classical" numerical distance effect: His RTs decreased as a function of the numerical distance from the internal reference $\left(r^{2}=.77, p<.001\right)$, exactly as in the control subjects and not as a function of the geometrical distance, measured in centimetres from one of S.W.'s drawings $\left(r^{2}=\right.$ $.13, p=0.29$; see Figure 3). If the distance effect was determined by straight-line geometrical distance on his number form, S.W.'s responses to 31 and 32 should be slower than those to 24 and 25 , as on his line 31 and 32 are closer to 28 than 24 and 25 are. However, this was not the case: He was faster comparing 31 and 32 with 28 (596 and $598 \mathrm{~ms}$ ) than he was comparing 24 or 25 (615 and $610 \mathrm{~ms}$, respectively), exactly as the controls. Of course, it remains possible that S.W.'s comparison times are determined by curvilinear distance along his number form, but this hypothesis is indistinguishable from the classical

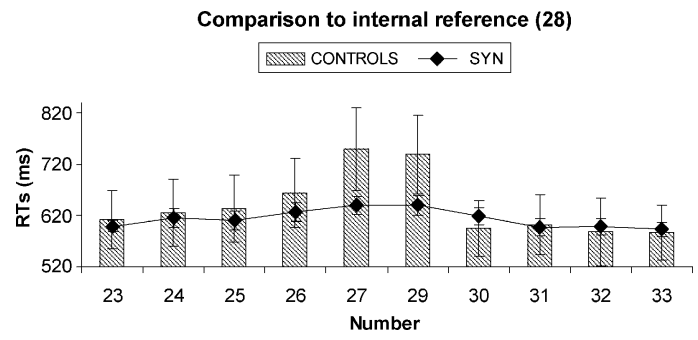

Figure 3. Plot of the control group's and subject S.W.'s mean RTs in the number comparison experiment. S.W. showed a normal distance effect. Errors bars give the standard error of the mean across subjects for the control group and the standard error across trials for subject S.W. numerical distance effect. Interestingly, despite the fact that S.W.'s RTs were modulated by numerical distance, the size of this distance effect (calculated as the slope of the linear regression between the five numerical distances and the $\mathrm{RTs}$ ) was smaller in S.W. than in the control group (ST: $p=.05$ ).

\section{Experiment 3: Comparison of numbers drawn on S.W.'s number line}

In order to investigate the automaticity of access to the number form, we investigated whether presenting drawings of S.W.'s number line on the computer screen could interfere with his performance in a number comparison task.

We presented pairs of digits positioned on a curved line for numbers $1-40$, redrawn by the experimenter on the basis of S.W.'s drawings. We asked S.W. and 10 control subjects to press the button located on the side of the larger number (left or right), with the instructions to maximize both speed and accuracy. The displays remained on screen until a response was detected. Each display could be "compatible" with S.W.'s number line (i.e., the two digits were placed at the correct locations according to his drawing), or "incompatible" (the two locations were inverted; see Figure 4A). The digits spanned from 1 to 40 and were presented in pairs representing a numerical distance of either 15 (i.e., 1-16, 25-40) or 23 (i.e., 2-25).

S.W. was extremely troubled by the presentation of numbers on a drawing of his number form, especially when the numbers were misplaced. He reported a strong feeling of discomfort throughout the entire testing session, and this was reflected in very slow response times relative to controls $(1,380 \mathrm{~ms}$ vs. $788 \mathrm{~ms}, \mathrm{ST}: p<.01)$ (see Figure 4A). Importantly, he was slower on incompatible than on compatible trials $(1,494 \mathrm{~ms}$ and $1,265 \mathrm{~ms}$, respectively), which was not the case for controls (here and elsewhere we used a one-tailed revised standardized difference test, RSDT, to test whether the difference between an individual's scores on two conditions is significantly different from the differences observed in a 
A

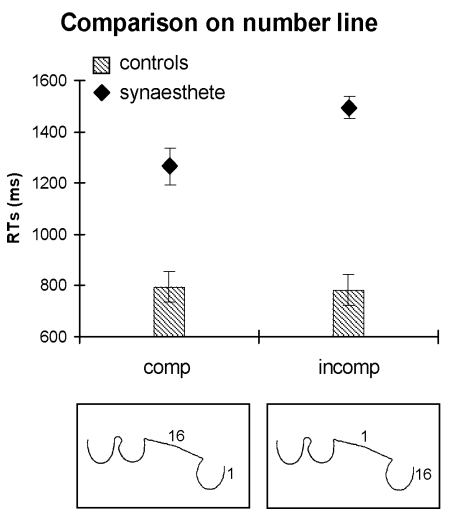

B

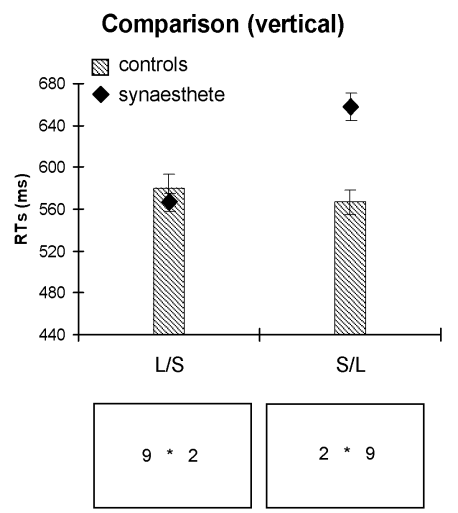

C

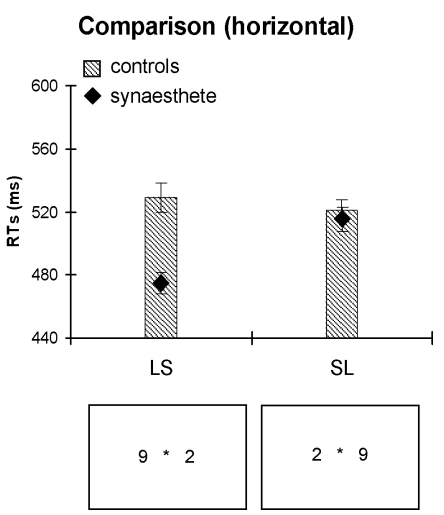

Figure 4. Plots of the control group's and S.W.'s mean RTs in the number comparison experiments. Pairs of digits were presented either on a drawing of the number line $(A)$, or simply horizontally $(B)$ or vertically $(C)$ around the fixation point. In all three experiments subject $S . W$. behaved differently from the control group, in that he was faster when the digits were presented in spatial layouts compatible with the global orientation of his number line (small numbers on the right and bottom). $S L=$ small number on the left; $L S=$ small number on the right; $S / L$ $=$ small number on top; $L / S=$ small number on the bottom. Errors bars give the standard error of the mean across subjects for the control group and the standard error across trials for subject S.W.

control sample; Crawford, Garthwaite, \& Gray, 2003; RSDT: $p=.002$ ).

\section{Comparison of numbers presented vertically or horizontally}

In order to explore further whether the numberspace associations were automatically triggered, even when the number form itself was not present on screen, we asked S.W. and 10 controls to compare the magnitude of pairs of numbers, which, in different blocks, were presented symmetrically either horizontally or vertically around a central fixation point.

Stimuli were pairs of Arabic numerals presented in white on a black background, $4 \mathrm{~cm}$ peripheral to a central fixation cross at a viewing distance of approximately $40 \mathrm{~cm}$ (5.7 degrees of visual angle), either vertically or horizontally, in two separate blocks. All 10 pairs with a distance of 2 from 1 to 12 were presented 16 times in each block. Participants were instructed to maintain fixation and to press on the side of the larger number, maximizing both speed and accuracy. The displays disappeared after they gave their answer.
Results showed that both the horizontal and the vertical spatial layout of the stimuli strongly influenced S.W.'s performance. In both cases S.W. was much faster with the congruent presentation order, whereas the controls showed no significant difference, thus resulting in a significant difference between S.W. and the controls (RSDT: $p<.01$, and $p<.05$, for the horizontal and the vertical layouts, respectively). For pairs of numbers presented vertically, S.W. was slower than controls when the larger number was on the bottom (ST: $p<.05)$, consistent with his bottom-to-top oriented number form (see Figure 4B). Even more interestingly, when numbers were displayed horizontally, S.W. was faster than the controls when the small number was presented on the right and the large number on the left (ST: $p<$ $.05)$, compatibly with his right-to-left oriented number form (Figure 4C).

\section{Parity judgement}

Finally, we tested S.W. and 10 controls on a parity judgement task, which was previously used to reveal implicit spatial-numerical associations (known as the SNARC effect: Spatial-Numerical 
Association of Response Codes; Dehaene et al., 1993). A single digit between 1 and 9 (except 5) was presented on the screen for $200 \mathrm{~ms}$, after the presentation of a fixation cross. Participants had to respond whether the digit was odd or even by pressing a button according to instructions, which were reversed halfway through the experiment. This task was performed under two different conditions (the order of which was counterbalanced across subjects): In one condition the stimuli were presented in the centre of the screen, while in the other they were presented in periphery, either to the left or to the right of the fixation cross. One participant's data from the central presentation condition was subsequently discarded as he did not understand the instructions.
In this task, S.W. performed like the controls. For each participant we calculated the slope of the linear regression between the difference in RT between right and left key presses and the number that was presented. For the peripheral presentation, the group mean slope was $-9.0 \mathrm{~ms}$ per number, indicating a superiority of the left hand for judging small numbers and a superiority of the right hand with larger numbers (SNARC, $\left.r^{2}=.75, p<.01\right)$. For S.W.'s data, although the linear regression did not quite reach significance $\left(r^{2}=.33, p=.13\right)$, the SNARC slope of $-12.3 \mathrm{~ms}$ was in the same direction and did not differ from the one observed in the control group (ST: $p=.37$; see Figure $5 \mathrm{~A}$, left panel). Indeed, S.W.'s slope was well within

\section{A}

Snarc Effect (peripheral presentation)

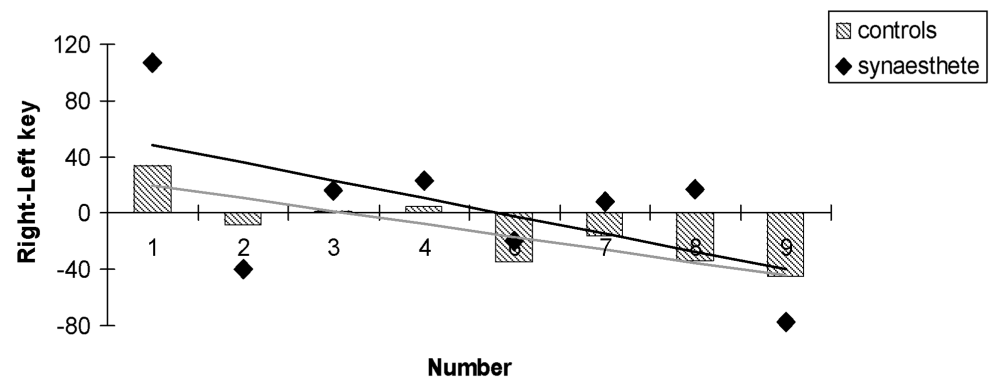

INDIVIDUAL SNARC (peripheral) REGRESSION SLOPES

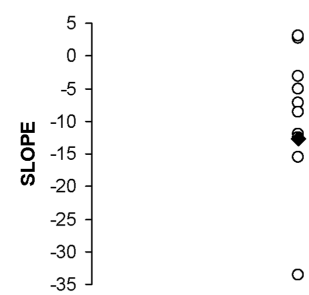

B Snarc Effect (central presentation)
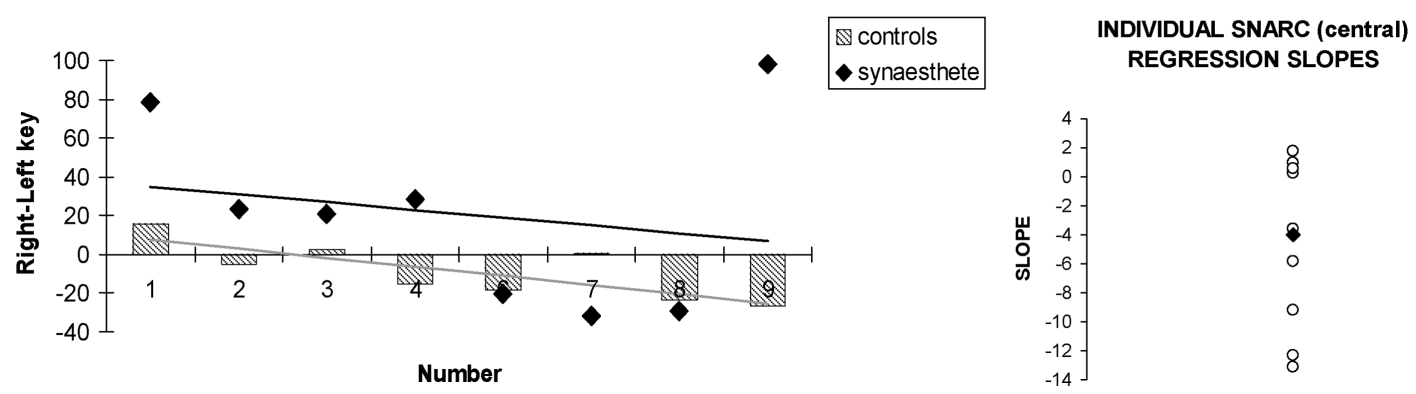

Figure 5. Plots of the SNARC effect. Subjects performed a parity judgement on single digits (1 to 4 and 6 to 9), presented either laterally to a central fixation cross $(A)$, or centrally (B). In the left panels, for each presented number, we plot the difference between the mean $R T$ s of the right and of the left key presses, for both the controls and $S$. W. This difference indicates the implicit association between numbers and space: Positive values indicate faster $R T$ s with the left hand, while negative values indicate faster RTs with the right hand. In the right panel, each data point represents the SNARC slope for one subject. The control subjects' data are represented with empty circles and $S$. W. with filled diamonds. 
the range of the control subjects and in fact more negative than the slope of 8 out of 10 control subjects (see Figure 5A, right panel). The results were similar for the central presentation (the slope for S.W. was more negative than the slope of 5 out of 9 control subject), where the group mean slope was $-4.6 \mathrm{~ms}$ per number (SNARC, $r^{2}=$ $.62, p<.05)$, and S.W.'s slope was $-3.9 \mathrm{~ms}$, a nonsignificant difference (ST: $p=.46$ ) although S.W.'s regression itself did not reach significance $\left(r^{2}=.04, p=.63\right.$; see Figure $\left.5 \mathrm{~B}\right)$.

\section{DISCUSSION}

We have described the spatial representation of numbers (number form) experienced by an individual (S.W.) and have demonstrated the existence of several objective correlates of this subjective number-space association. Two findings suggest that the number-form synaesthesia in S.W. is a genuine phenomenon. First, his spatial representation of numbers is highly consistent over testing sessions separated by a delay of two years. Second, it interferes with chronometric performance: Compared to a group of control subjects, S.W. was faster in comparing the magnitude of pairs of digits when the spatial layouts of the stimuli were compatible with the global orientation of his number line (small numbers presented on the right and bottom, and large numbers presented on the left and top). Compared to the control group, he was slower at comparing pairs of digits when the smaller digit appeared at the top and the larger at the bottom, and he was actually faster when the smaller was on the right and the larger on the left. The latter facilitation effect of more than $40 \mathrm{~ms}$ (which allowed him to respond very quickly, with a mean RT of $470 \mathrm{~ms}$ ) suggests a fast activation of the spatial representation of numbers in S.W. Compared to this facilitatory effect, one should not interpret the slowing for the (incongruent) vertical layout as necessarily reflecting an inhibitory process. In fact, in a context of general slowness ${ }^{1}$ for the vertical condition layout, S.W. still shows a significant compatibility effect.

Taken together, these findings suggest that numerals, at least in a number comparison context, obligatorily trigger a genuine sense of space in subject S.W. Even for nonsynaesthetic subjects, numbers are thought to be internally represented on a spatially oriented mental "number line". Evidence that the concept of mental number line is more than a metaphor comes from behavioural and brain lesion studies (Dehaene et al., 1993; Fischer et al., 2003; Restle, 1970; Vuilleumier, Ortigue, \& Brugger, 2004; Zorzi, Priftis, \& Umiltà, 2002). This research has shown that close numbers are less discriminable than numbers that are far apart (distance effect) and that small numbers are associated with the left side of the space and large numbers with the right side (SNARC effect; attention priming effect; number bisection bias in hemineglect). We therefore took advantage of the particular spatial characteristics of the number form experienced by S.W. (right-to-left oriented and curvilinear) to investigate the nature of the number representation from which these effects originate. Surprisingly, neither the distance nor the SNARC effects were modulated by S.W.'s particular spatial representation of numbers. The fact that S.W. presented an unchanged numerical distance effect in number comparison suggests that this effect is not generated by an explicit spatial representation of numbers. Together with the observation that judgements over virtually all physical continua show similar distance effects, this would imply that the distance effect is a very general signature of magnitude-based decisions and not necessarily linked to spatial

\footnotetext{
${ }^{1}$ S.W., as the control subjects, responded with his hands vertically aligned in the vertical condition: The right hand was placed on top (on the key "6" of a QWERTY keyboard) and the left hand on the bottom (key "b" of a QWERTY keyboard). S.W. is a violin player, for which the most natural position of his hands in vertical space is left above right (as for holding and playing the instrument). It is possible therefore that the general slowing that we observe in S.W. is due to the incongruence between the hand position that would have been natural for him and the position that was required by the experimenter.
} 
representations (Fias, Lammertyn, Reynvoet, Dupont, \& Orban, 2003; Pinel, Piazza, Le Bihan, \& Dehaene, 2004).

Moreover, S.W. presents an unchanged rather than a reversed SNARC effect when judging the parity of single digits. This dissociation between subjective and objective measures indicates that the presence of an implicit association between small numbers and the left side of the space can coexist with a conscious experience of an opposite spatial representation of the number sequence (S.W.'s number form is right-to-left oriented). The number-space associations at the origin of the SNARC effect as measured in parity judgement tasks are likely to be linked to the direction of writing as, for example, Iranian or Lebanese subjects who are used to reading from right to left tend to exhibit an inverse SNARC effect (Dehaene et al., 1993; Zebian, 2005). However, the intersubject variability of the SNARC effect is also associated to the interindividual differences in visuospatial abilities: In a group of children from 7 to 12 years old for whom an overall normal SNARC effect could be observed, approximately $30 \%$ of the subjects did not show a negative slope. Furthermore, in a group of children of the same age presenting visuo-spatial deficits, $60 \%$ did not show a classical SNARC slope (Bachot, Gevers, Fias, \& Roeyers, 2005). Overall, a larger SNARC effect was observed in subjects with better visuospatial scores. It is possible that subjects with low visuo-spatial abilities fail to impose strong spatial constraints to the mental sequence of numbers during development, and this would result in the absence of a strong numerical-spatial effect. If this scenario was correct, one might predict that number-form synaesthetes exhibit stronger visuo-spatial abilities than do control subjects.

The extent to which number-form synaesthesia depends on the same mechanisms as the numerical-spatial interactions described in nonsynaesthetic observers remains an open question. It seems plausible that both number-form synaesthesia and the more implicit and universal spatial-numerical associations arise from brain mechanisms of cross-activation between spatial and numerical representations in the parietal lobe
(E. M. Hubbard, Piazza, Pinel, \& Dehaene, 2005b). Speculatively, it seems likely that the cortical proximity between coding of space and numerical quantity in the parietal lobe (Piazza, Izard, Pinel, Le Bihan, \& Dehaene, 2004; Simon, Mangin, Cohen, Le Bihan, \& Dehaene, 2002) makes the development of number forms particularly natural in children during their acquisition of numerical symbols. Interestingly, in support for a crucial role of parietal cortex in number-form synaesthesia, Spalding and Zangwill (1950) described a number-form synaesthetic patient who suffered a gunshot wound (which entered near the right angular gyrus and lodged near the left temporal-parietal junction) and who thereafter complained that his synaesthetic number form was no longer distinct. Crosstalk between spatial and numerical representations within the parietal cortex might occur because of transitory exuberant synaptic connections (Baron-Cohen, Harrison, Goldstein, \& Wyke, 1993; E. M. Hubbard et al., 2005a; Paulesu et al., 1995) or because the representation of numbers expands during arithmetic learning and invades other territories initial devoted to spatial representation (Dehaene, 1997).

Parietal cortex encompasses different spatial frames: for instance, head centred, eye centred, or object centred (Colby, 1998; Colby \& Goldberg, 1999). Thus, one might ask which spatial frame of reference generates both synaesthetic and normal numerical-spatial interactions. In the number domain, different studies of the SNARC effect have shown that it is possible to bias subjects to use one spatial reference frame rather than another-for example, by asking them to think of numbers as situated either on a clock-face or on a horizontal line as on the $x$-axis of the Cartesian plane (Bachtold, Baumuller, \& Brugger, 1998; Vuilleumier et al., 2004). It is possible that numbers have an easier access to such different spatial frames in synaesthesia, and this facilitation might generate conjoint but sometimes conflicting multiple spatial representations of numbers. For example, parietal activation related to numbers could sometimes spread towards the posterior intraparietal cortex, where 
an eye-centred representation of space exists, laterally in head-centred regions, or anteriorly in regions containing hand-centred representations of space.

The possible coexistence of multiple conflicting spatial frames in synaesthesia could accommodate our apparently contradictory findings of the presence of a "normal" SNARC effect (evidence of a left-to-right orientation of numbers) in a parity judgement task in which each number was presented individually on the screen and a reversed effect in comparison tasks where pairs of numbers were both presented on the screen. In the latter case it is possible that the combination of (a) the fact that the stimuli consisted of pairs of numbers both presented on the screen and (b) the fact that the task was a number comparison task, which directly requires access and exploration of the mental number line, created the best conditions to trigger the synaesthetic representation, rather than the most implicit "canonical" left-toright linear representation of numbers. On the contrary, for the parity judgement task, together with the fact that in such an experiment we presented one number at a time on the screen, the access to the number line may have been only implicit, and it is therefore possible that this allowed the emergence of the number-space associations that are generally implicit and shaped by the direction of writing (i.e., the classical "SNARC" effect). Indeed, evidence for dissociations between implicit and explicit number-space representations comes from a recent study of numerical bisection and the SNARC effect in neglect patients (Priftis, Zorzi, Meneghello, Marenzi, \& Umiltà, 2006). These patients were impaired on a number interval bisection task (a task directly requiring access and exploration of the mental number line), but presented a normal SNARC effect on a parity judgement task (a task that does not directly require the explicit manipulation of the number sequence).

In sum, our data support the idea that number forms, at least in S.W., are more intensely triggered whenever a strong notion of sequence is induced either by the task or by the stimuli.
Indeed, this is in agreement with the fact that individuals presenting visuo-spatial forms for numbers, just as S.W., very often also have forms for other ordinal sequences, such as days, months, or letters (Sagiv et al., in press).

Finally, why are some synaesthetic "number forms" so similar across subjects separated by more than a century? Although the constraints that structure number forms remain mysterious, the recurrence of culturally driven themes (the clock face and the boundaries at decades), suggests that culture has an important influence on the shaping of number forms in synaesthesia, exactly as is the case for the nonsynaesthetic number line whose direction is shaped by the direction of writing. Further research on a large number of subjects will be needed to investigate the interactions between neurological differences and cultural constraints in the ontogenesis of spatial-numerical associations.

Manuscript received 21 June 2005 Revised manuscript received 24 April 2006 Revised manuscript accepted 28 April 2006 First published online 15 July 2006

\section{REFERENCES}

Bachot, J., Gevers, W., Fias, W., \& Roeyers, H. (2005). Number sense in children with visuospatial disabilities: Orientation of the mental number line. Psychology Science, 47, 172-183.

Bachtold, D., Baumuller, M., \& Brugger, P. (1998). Stimulus-response compatibility in representational space. Neuropsychologia, 36, 731-735.

Baron-Cohen, S., Harrison, J., Goldstein, L. H., \& Wyke, M. (1993). Coloured speech perception: Is synaesthesia what happens when modularity breaks down? Perception, 22, 419-426.

Baron-Cohen, S., Wyke, M. A., \& Colin, B. (1987). Hearing words and seeing colours: An experimental investigation of a case of synaesthesia. Perception, 16, 761-767.

Calkins, M. W. (1892). A statistical study of pseudochromesthesia and of mental-forms. American Journal of Psychology, 5, 439-464.

Colby, C. L. (1998). Action-oriented spatial reference frames in cortex. Neuron, 20, 15-24. 
Colby, C. L., \& Goldberg, M. E. (1999). Space and attention in parietal cortex. Annual Review of Neuroscience, 22, 319-349.

Crawford, J. R., \& Howell, D. C. (1998). Comparing an individual's test score against norms derived from small samples. The Clinical Neuropsychologist, 12, 482-486.

Crawford, J. R., Garthwaite, P.H., \& Gray, C. D. (2003). Wanted: Fully operational definitions of dissociations in single-case studies. Cortex, 39, 357-370.

Cytowic, R. E. (2002). Synaesthesia: A union of the senses (2nd ed.). Cambridge, MA: MIT Press.

Dehaene, S. (1997). The number sense. New York: Oxford University Press.

Dehaene, S. (2002). Single-neuron arithmetic. Science, 297, 1652-1653.

Dehaene, S., Bossini, S., \& Giraux, P. (1993). The mental representation of parity and numerical magnitude. Journal of Experimental Psychology: General, 122, 371-396.

Dehaene, S., Dupoux, E., \& Mehler, J. (1990). Is numerical comparison digital: Analogical and symbolic effects in two-digit number comparison. Journal of Experimental Psychology: Human Perception and Performance, 16, 626-641.

Dixon, M. J., Smilek, D., \& Merikle, P. M. (2004). Not all synaesthetes are created equal: Projector versus associator synaesthetes. Cognitive Affective and Bebavioral Neuroscience, 4, 335-343.

Fias, W., Lammertyn, J., Reynvoet, B., Dupont, P., \& Orban, G. A. (2003). Parietal representation of symbolic and nonsymbolic magnitude. Journal of Cognitive Neuroscience, 15, 47-56.

Fischer, M. H., Castel, A. D., Dodd, M. D., \& Pratt, J. (2003). Perceiving numbers causes spatial shifts of attention. Nature Neuroscience, 6, 555-556.

Galton, F. (1880a). Visualised numerals. Nature, 21, 252-256.

Galton, F. (1880b). Visualised numerals. Nature, 21, 494-495.

Hubbard, E. M., Arman, A. C., Ramachandran, V. S., \& Boynton, G. M. (2005a). Individual differences among grapheme-color synesthetes: Brain-behavior correlations. Neuron, 45, 975-985.

Hubbard, E. M., Piazza, M., Pinel, P., \& Dehaene, S. (2005b). Interactions between number and space in parietal cortex. Nature Reviews Neuroscience, 6, 435-448.

Hubbard, E. M., \& Ramachandran, V. S. (2005). Neurocognitive mechanisms of synesthesia. Neuron, $48,509-520$.
Hubbard, T. L. (1996). Synesthesia-like mappings of lightness, pitch, and melodic interval. American Journal of Psychology, 109, 219-238.

Köhler, W. (1920). Gestalt psychology. New York: Liveright.

Moyer, R. S., \& Landauer, T. K. (1967). Time required for judgements of numerical inequality. Nature, 215, 1519-1520.

Nieder, A., \& Miller, E. K. (2003). Coding of cognitive magnitude. Compressed scaling of numerical information in the primate prefrontal cortex. Neuron, 37, 149-157.

Palmeri, T. J., Blake, R., Marois, R., Flanery, M. A., \& Whetsell, W., Jr. (2002). The perceptual reality of synesthetic colors. Proceedings of the National Academy of Sciences of the United States of America, 99, 4127-4131.

Paulesu, E., Harrison, J., Baron-Cohen, S., Watson, J. D., Goldstein, L., Heather, J., et al. (1995). The physiology of coloured hearing. A PET activation study of colour-word synaesthesia. Brain, 118, 661-676.

Piazza, M., Izard, V., Pinel, P., Le Bihan, D., \& Dehaene, S. (2004). Tuning curves for approximate numerosity in the human intraparietal sulcus. Neuron, 44, 547-555.

Pinel, P., Piazza, M., Le Bihan, D., \& Dehaene, S. (2004). Distributed and overlapping cerebral representations of number, size, and luminance during comparative judgments. Neuron, 41, 983-993.

Priftis, K., Zorzi, M., Meneghello, M., Marenzi, R., \& Umiltà, C. (2006). Explicit vs. implicit processing of representational space in neglect: Dissociations in accessing the mental number line. Journal of Cognitive Neuroscience, 18, 680-688.

Ramachandran, V. S., \& Hubbard, E. M. (2001a). Psychophysical investigations into the neural basis of synaesthesia. Proceedings of the Royal Society of London Series B: Biological Sciences, 268(1470), 979-983.

Ramachandran, V. S., \& Hubbard, E. M. (2001b). Synaesthesia- a window into perception, thought and language. Journal of Consciousness Studies, 8, 3-34.

Restle, F. (1970). Speed of adding and comparing numbers. Journal of Experimental Psychology, 83, 274-278.

Rich, A. N., \& Mattingley, J. B. (2002). Anomalous perception in synaesthesia: A cognitive neuroscience perspective. Nature Reviews Neuroscience, 3, 43-52.

Sagiv, N., Simner, J., Collins, J., Butterworth, B., \& Ward, J. (in press). What is the relationship between synaesthesia and visuo-spatial number forms? Cognition. 
Seron, X., Pesenti, M., Noël, M. P., Deloche, G., \& Cornet, J.-A. (1992). Images of numbers or when 98 is upper left and 6 sky blue. Cognition, 44, 159-196.

Shepard, R. N., Kilpatrick, D. W., \& Cunningham, J. P. (1975). The internal representation of numbers. Cognitive Psychology, 7, 82-138.

Simon, O., Mangin, J. F., Cohen, L., Le Bihan, D., \& Dehaene, S. (2002). Topographical layout of hand, eye, calculation, and language-related areas in the human parietal lobe. Neuron, 33, 475-487.

Smilek, D., Dixon, M. J., Cudahy, C., \& Merikle, P. M. (2001). Synaesthetic photisms influence visual perception. Journal of Cognitive Neuroscience, 13, 930-936.
Spalding, J. M. K., \& Zangwill, O. L. (1950). Disturbance of number-form in a case of brain injury. Journal of Neurology, 13, 24-29.

Vuilleumier, P., Ortigue, S., \& Brugger, P. (2004). The number space and neglect. Cortex, 40, 399-410.

Zebian, S. (2005). Linkages between number concepts, spatial thinking, and directionality of writing: The SNARC effect and the REVERSE SNARC effect in English and Arabic monoliterates, biliterates, and illiterate Arabic speakers. Journal of Cognition and Culture, 5, 165-190.

Zorzi, M., Priftis, K., \& Umiltà, C. (2002). Brain damage: Neglect disrupts the mental number line. Nature, 417(6885), 138-139. 